\title{
Endoscopic ultrasonography for differential diagnosis of polypoid gall bladder lesions: analysis in surgical and follow up series
}

\author{
M Sugiyama, Y Atomi, T Yamato
}

\begin{abstract}
Background-Differential diagnosis is often difficult for small $(\leqslant 20 \mathrm{~mm})$ polypoid lesions of the gall bladder.

Aim-To assess the diagnostic accuracy of endoscopic ultrasonography (EUS) for polypoid lesions in a surgical and follow up series.

Methods-A total of 194 patients with small polypoid lesions underwent both ultrasonography and EUS. A tiny echogenic spot or an aggregation of echogenic spots and multiple microcysts or a comet tail artefact indicated cholesterol polyp and adenomyomatosis respectively. Other lesions were diagnosed as neoplastic (adenoma or adenocarcinoma). In the 58 patients who underwent surgery, the histological diagnoses were cholesterol polyp ( $\mathbf{n}=36)$, adenomyomatosis $(\mathbf{n}=7)$, adenoma $(n=4)$, and adenocarcinoma (n $=11)$. Of the remaining 136 patients with an EUS diagnosis of non-neoplastic lesions, 125 were followed up with ultrasonography alone or with EUS for 1-8.7 years (mean 2.6 years).
\end{abstract}

Results-In the surgical series, EUS (97\%) differentiated polypoid lesions more precisely than ultrasonography $(76 \%)$. During follow up, the lesions remained unchanged in size in $109(87 \%)$ of the 125 patients with non-neoplastic lesions diagnosed by EUS. No neoplastic lesions developed in these patients. Ultrasonography had shown lesions to be neoplastic in $13 \%$ of the follow up series.

Conclusions-EUS is highly accurate for differentially diagnosing polypoid gall bladder lesions. It is recommended when ultrasonography cannot rule out neoplastic lesions. Non-neoplastic lesions diagnosed by EUS may be followed and observed with ultrasonography.

(Gut 2000;46:250-254)

Keywords: gall bladder; polypoid lesion; cholesterol polyp; ultrasonography; endoscopic ultrasonography

Third Department of Internal Medicine T Yamato

Correspondence to: Dr M Sugiyama, First Department of Surgery, Kyorin University School of Medicine 6-20-2 Shinkawa, Mitaka, Tokyo 181-8611, Japan.

Accepted for publication 18 August 1999 neoplastic (adenoma and adenocarcinoma). ${ }^{3}$ Cholesterol polyps are the most common type of polypoid lesion and have no malignant potential. Typically, on ultrasonography, cholesterol polyps are small $(\leqslant 10 \mathrm{~mm})$ echogenic pedunculated masses without acoustic shadowing. ${ }^{6-10}$ However, some cholesterol polyps, particularly those larger than $10 \mathrm{~mm}$, appear partially or completely echopenic on ultrasonography. ${ }^{9}{ }^{10}$ Such polyps are difficult to distinguish from adenocarcinomas. Adenocarcinomas are uncommon but should be precisely differentiated from non-neoplastic lesions because some small polypoid carcinomas can be curatively resected. Therefore an imaging modality that allows accurate differential diagnosis is required.

We previously reported the high accuracy of endoscopic ultrasonography (EUS) in differentiating polypoid gall bladder lesions in a surgical series. ${ }^{9} 10$ In principle, non-neoplastic lesions prospectively diagnosed by EUS have been followed up instead of being excised in our department. Herein, the diagnostic accuracy of EUS for polypoid gall bladder lesions is assessed in a follow up series as well as a surgical series.

Patients and methods

Between 1988 and 1997, 194 consecutive patients underwent EUS for small $(\leqslant 20 \mathrm{~mm}$ in maximum diameter) polypoid lesions of the gall bladder which had been detected by transabdominal ultrasonography (89 men and 105 women with a mean age of 52 (range 22-81) years). Of the 194 patients, 131 were referred for further evaluation with EUS from other institutions, mainly because the doctors could not rule out neoplastic lesions on ultrasonography. In the 63 remaining patients, the polypoid lesions were first detected by ultrasonography in our department. In principle, EUS was indicated for polypoid lesions exceeding $5 \mathrm{~mm}$ or those suspected of neoplasia. Forty eight patients were symptomatic. In most of the other patients, the lesions were detected incidentally by ultrasonography during a regular health check or preoperative screening for other diseases. The interval between ultrasonography and EUS ranged from 0 to 25 days (mean 10 days). The maximum diameter of the polypoid lesions was determined ultrasonographically. In patients with multiple polyps, the size of the largest polyp was measured.

Abbreviation used in this paper: EUS, endoscopic ultrasonography. 
Transabdominal ultrasonography was performed using a real time scanner with a 3.5 MHZ linear array or curved array transducer (SAL-77A or SSA-270A; Toshiba, Tokyo, Japan; or SSD-650 or SSD-2000; Aloka, Tokyo, Japan). EUS was performed using an echoendoscope with a $7.5 \mathrm{MHZ}$ rotating transducer (GF-UM2/EU-M2, GF-UM3/EUM3, or GF-UM200/EU-M30; Olympus, Tokyo, Japan). The gall bladder was visualised from the duodenum and the gastric antrum. A balloon filled with $5-15 \mathrm{ml}$ water was used to provide acoustic coupling. For sedation, 5-10 mg diazepam was administered intravenously.

Data on ultrasonography and EUS were obtained from prospective official reports. EUS was performed by one of the authors who had knowledge of the ultrasonographic findings. The previously reported ultrasonographic and EUS criteria for differential diagnosis of polypoid lesions were as follows. ${ }^{9}{ }^{10}$ (a) Cholesterol polyps show an internal echo pattern characterised as a tiny echogenic spot (entire lesion appearing as a single $1-5 \mathrm{~mm}$ spot that is homogeneously and highly echogenic) or an aggregation of multiple highly echogenic 1-3 $\mathrm{mm}$ spots with or without echopenic areas (fig 1). (b) Adenomyomatosis (localised type) ${ }^{3}$ is imaged as a sessile echogenic mass containing multiple microcysts (usually composed of 2-8 $\mathrm{mm}$ cysts) or a comet tail artefact (V-shaped reverberation ultrasound artefact) ${ }^{78}$ (fig 2). (c) In the absence of echogenic spots, multiple microcysts, or a comet tail artefact, these lesions are diagnosed as neoplastic (adenocarcinoma or adenoma) (figs 3 and 4). Sessile lesions suggest malignancy.

All patients with suspected neoplastic lesions on EUS underwent surgery. In principle, surgery was not indicated for patients with an EUS diagnosis of non-neoplastic lesions, except for symptomatic cases or those undergoing combined operation for other abdominal diseases. Non-surgical cases were followed up, once or twice a year, by ultrasonography alone or with EUS. In our surgical series, the ultrasonographic and EUS diagnosis was compared with the histopathological diagnosis. In the follow up cases, changes in sonographic findings were investigated. Changes in size exceeding $3 \mathrm{~mm}$ on ultrasonography were defined as enlarged or reduced.

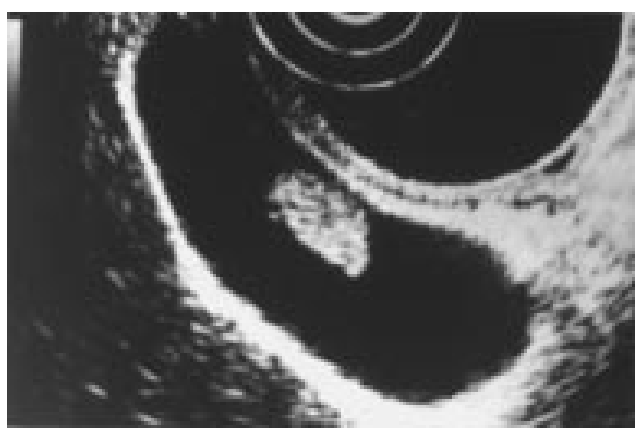

Figure 1 Cholesterol polyp of the gall bladder. Endoscopic ultrasonography shows a $12 \mathrm{~mm}$ granular surfaced pedunculated mass which has an internal echo pattern characterised by an aggregation of echogenic spots. Histological examination of the surgical specimen showed a cholesterol polyp.

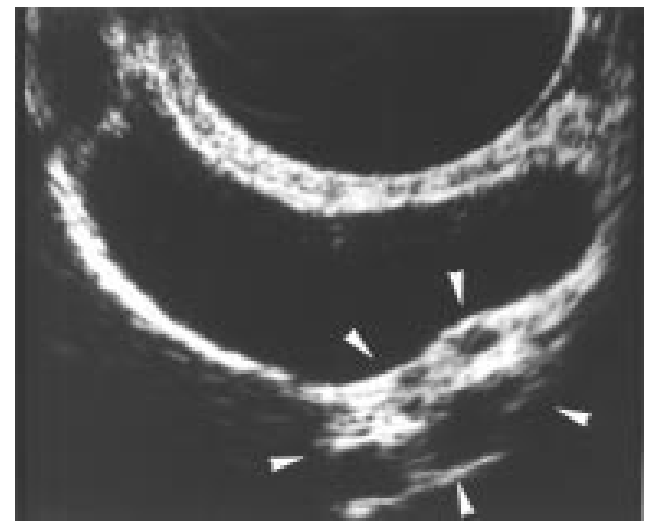

Figure 2 Adenomyomatosis of the gall bladder. Endoscopic ultrasonography shows a $20 \mathrm{~mm}$ smooth-surfaced sessile mass (arrowheads) with multiple microcysts. Histological examination confirmed adenomyomatosis.

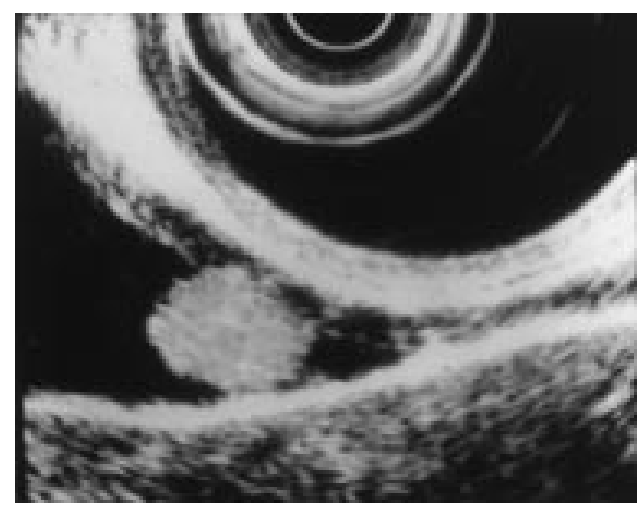

Figure 3 Adenoma of the gall bladder. Endoscopic ultrasonography shows a $14 \mathrm{~mm}$ granular surfaced homogeneously echogenic pedunculated mass. Histological diagnosis was adenoma.

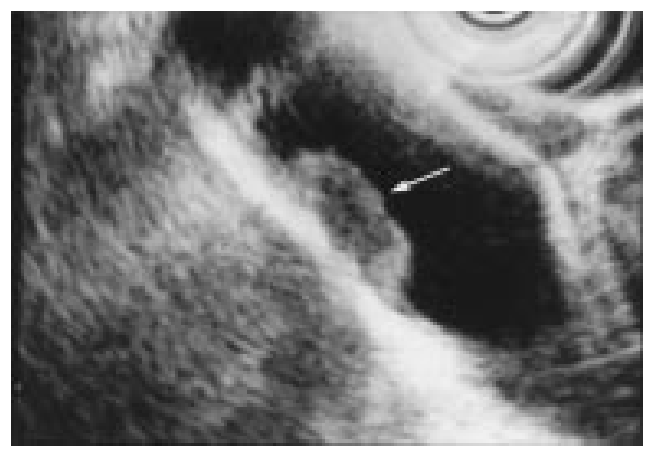

Figure 4 Adenocarcinoma of the gall bladder. Endoscopic ultrasonography shows a $19 \mathrm{~mm}$ smooth surfaced heterogeneously echogenic sessile mass (arrow). Histological examination of the surgical specimen showed

adenocarcinoma invading the subserosal layer of the gall bladder.

Informed consent was obtained from all patients. Results were analysed by Fisher's exact probability test or the Wilcoxon test where appropriate. Differences were considered significant when $\mathrm{p}<0.05$.

\section{Results}

In all 194 patients, the gall bladder was adequately visualised at EUS. The sizes of the polypoid lesions measured ultrasonographically approximated (within $2 \mathrm{~mm}$ ) those measured at EUS. The initial EUS diagnosis of 
polypoid gall bladder lesions included cholesterol polyp ( $\mathrm{n}=158)$, adenomyomatosis (localised type) ( $n=19)$, and neoplastic lesions $(\mathrm{n}=17)$.

\section{SURGICAL SERIES}

Of the 194 patients, 58 underwent cholecystectomy within one month of the EUS examination. Histological examination of the polypoid lesions disclosed cholesterol polyps in 36, adenomyomatosis in seven, adenoma in four, and adenocarcinoma in 11 patients (table 1). The lesion sizes measured after cholecystectomy approximated (within $2 \mathrm{~mm}$ ) those measured ultrasonographically. The prevalence of neoplastic lesions was $0 \%$ in $1-5 \mathrm{~mm}, 19 \%$ in $6-10 \mathrm{~mm}, 33 \%$ in $11-15 \mathrm{~mm}$, and $55 \%$ in 16-20 mm lesions. Before surgery, EUS had shown the polypoid lesions to be cholesterol polyps $(n=34)$, adenomyomatosis $(n=7)$, or neoplastic lesions $(n=17)$ (table 2$)$. EUS and ultrasonography correctly distinguished among polypoid lesions in $56(97 \%)$ and $44(76 \%)$ of 58 patients respectively; the difference was significant $(\mathrm{p}<0.01)$. In 12 of 36 patients with histologically confirmed cholesterol polyps, ultrasonography depicted a homogeneously or heterogeneously echogenic or entirely echopenic mass without aggregation of echogenic spots, which led to misdiagnosis of neoplastic

Table 1 Histological diagnosis and size of polypoid gall bladder lesions in the surgical series

\begin{tabular}{llllll}
\hline Size $(\mathrm{mm})$ & Cholesterol polyp & Adenomyomatosis & Adenoma & Adenocarcinoma & Total \\
\hline $1-5$ & 11 & 0 & 0 & 0 & 11 \\
$6-10$ & 15 & 2 & 1 & 3 & 21 \\
$11-15$ & 7 & 3 & 2 & 3 & 15 \\
$16-20$ & 3 & 2 & 1 & 5 & 11 \\
Total & 36 & 7 & 4 & 11 & 58 \\
\hline
\end{tabular}

Table 2 EUS and ultrasonographic diagnosis of polypoid gall bladder lesions in the surgical series

\begin{tabular}{lrll}
\hline & \multicolumn{2}{l}{ Pathological diagnosis } & \\
\cline { 2 - 4 } & Cholesterol polyp & Adenomyomatosis & Neoplastic lesion \\
\hline EUS diagnosis & & & \\
$\quad$ Cholesterol polyp & 34 & 0 & 0 \\
$\quad$ Adenomyomatosis & 0 & 7 & 0 \\
$\quad$ Neoplastic lesion & 2 & 0 & 15 \\
Ultrasonographic diagnosis & 24 & 0 & 0 \\
$\quad$ Cholesterol polyp & 0 & 5 & 0 \\
$\quad$ Adenomyomatosis & 12 & 2 & 15 \\
$\quad$ Neoplastic lesion & & & \\
\hline
\end{tabular}

Table 3 Endoscopic ultrasonographic (EUS) and ultrasonographic diagnosis of polypoid gall bladder lesions in the follow up series

\begin{tabular}{llllr}
\hline \multirow{4}{*}{ EUS diagnosis } & \multicolumn{4}{l}{ Ultrasongraphic diagnosis } \\
\cline { 2 - 5 } & Cholesterol polyp & Adenomyomatosis & Neoplastic lesion & Total \\
\hline Cholesterol polyp & 100 & 0 & 14 & 114 \\
Adenomyomatosis & 0 & 9 & 2 & 11 \\
Neoplastic lesion & 0 & 0 & 0 & 0 \\
Total & 100 & 9 & 16 & 125 \\
\hline
\end{tabular}

Table 4 Change in size of polypoid gall bladder lesion in the follow up series

\begin{tabular}{llllll}
\hline Initial size (mm) & Enlarged & Unchanged & Reduced & Disappeared & Total \\
\hline $1-5$ & 4 & 25 & 0 & 2 & 31 \\
$6-10$ & 4 & 65 & 3 & 0 & 72 \\
$11-15$ & 1 & 13 & 1 & 0 & 15 \\
$16-20$ & 0 & 6 & 1 & 0 & 7 \\
Total & 9 & 109 & 5 & 2 & 125 \\
\hline
\end{tabular}

lesions. In 10 of these 12 patients, EUS showed an aggregation of echogenic spots and correctly diagnosed cholesterol polyp. Although ultrasonography showed multiple microcysts and/or a comet tail artefact in only five of seven patients with histologically confirmed adenomyomatosis, EUS showed such findings in all seven. Ten patients had concurrent gall bladder stones.

\section{FOLLOW UP SERIES}

Of the 194 patients, 136 with an EUS diagnosis of non-neoplastic lesion did not undergo cholecystectomy during the first year of follow up. Nine patients with abdominal discomfort refused surgical treatment. The other patients were asymptomatic. Of these 136 patients, three died from non-biliary diseases 1.5-3 years later and eight were lost to follow up. The 125 remaining patients were followed up by ultrasonography, once or twice a year, for 1-8.7 years (mean 2.6 years). These 125 patients constituted the follow up series. Of the 125 patients, 34 underwent follow up EUS, mainly for polypoid lesions which had been $\geqslant 10 \mathrm{~mm}$ on the initial examination or were enlarged during follow up.

The initial EUS had shown the polypoid lesions to be cholesterol polyps ( $n=114$ ) or adenomyomatosis $(n=11)$ in all 125 patients in the follow up series (table 3). In $16(13 \%)$ of the 125 patients, ultrasonography had indicated neoplastic lesions for similar reasons to those in the surgical series. The polypoid lesions were single in 45 and multiple in 69 of the 114 patients with cholesterol polyps diagnosed by EUS. All 11 lesions diagnosed as adenomyomatosis were single. The initial size of the lesion exceeded $10 \mathrm{~mm}$ in $22(18 \%)$ patients (table 4).

During the follow up period, there was no change in lesion size in $109(87 \%)$ of the 125 patients (table 4$)$. Nine patients $(7 \%)$ showed an increase in lesion size; the maximal increase was $4 \mathrm{~mm}$ ( $\mathrm{n}=2$; over 3.5 and 4.2 years respectively). No patients showed changes in the configuration or internal echo pattern of the polypoid lesions at either EUS or ultrasonography. In 109 patients in whom both initial ultrasonography and EUS had diagnosed polypoid lesions as non-neoplastic, follow up ultrasonography and EUS $(n=21)$ showed no findings suggesting neoplasia. In the 16 remaining patients in whom non-neoplastic lesions had been diagnosed by EUS alone, follow up ultrasonography showed no structural changes. Of the 16 patients, 13 also underwent the follow up EUS, which diagnosed the lesions as non-neoplastic. In all nine patients who showed an increase in lesion size, follow up EUS disproved neoplastic lesions. Three patients underwent cholecystectomy for polypoid lesions which had been diagnosed as cholesterol polyps based on the initial EUS, after a $2-5$ year observation period. The surgical indications were based on symptoms (13 mm lesion in one patient) or an increase in size (from 7 to $11 \mathrm{~mm}$ over a 3.5 year period in one patient; from 11 to $14 \mathrm{~mm}$ over a 2.5 year period in another). The latter two patients 
underwent follow up EUS three and two and a half years after the initial examination but showed no changes in the echo pattern of the polypoid lesions. Histological examination confirmed cholesterol polyp in all three patients.

\section{Discussion}

Among polypoid gall bladder lesions, a solitary lesion, a diameter greater than $10 \mathrm{~mm}$, a sessile appearance, low echogenicity, and rapid growth on ultrasonography have been reported to suggest adenocarcinoma. ${ }^{49-14}$ However, these ultrasonographic findings alone cannot definitely distinguish adenocarcinoma from non-neoplastic lesions. Although colour Doppler ultrasonography, enhanced computed tomography, and dynamic magnetic resonance imaging may facilitate differentiation of polypoid lesions by analysis of their vascularity, the diagnostic accuracy of these modalities remains unsatisfactory. ${ }^{15-17}$

We have previously reported that the echo pattern rather than the size of polypoid lesions is important in differential diagnosis. ${ }^{9}{ }^{10} \mathrm{~A}$ tiny echogenic spot or an aggregation of echogenic spots and multiple microcysts or a comet tail artefact are pathognomonic for cholesterol polyp and adenomyomatosis respectively. ${ }^{9}$ Polypoid lesions without such findings indicate neoplasia. An echogenic spot represents a mass of foamy histiocytes containing cholesterol, and an echopenic area corresponds to proliferation of glandular epithelia. ${ }^{9}$ Multiple microcysts and a comet tail artefact represent proliferation of Rokitansky-Aschoff sinus and intramural calculus respectively. ${ }^{7}$ EUS displays the fine structure of polypoid lesions more accurately than ultrasonography because the former provides images of higher resolution. In the present surgical series, EUS differentiated polypoid lesions more precisely than ultrasonography.

In our previous studies, EUS failed to differentiate reliably between adenomas and adenocarcinomas. ${ }^{9}{ }^{10}$ They showed only that, of these lesions, all the sessile ones were malignant. Adenomas are known to have a malignant potential (adenoma-carcinoma sequence). ${ }^{18} 19$ Because adenomas and adenocarcinomas both require surgical treatment, distinguishing between these lesions is not essential for their management.

Herein, we studied the natural history of polypoid gall bladder lesions in 125 patients in whom cholesterol polyp or adenomyomatosis had been diagnosed by EUS. During a mean follow up period of 2.6 years, all the lesions remained unchanged in ultrasonographic structure. Most (87\%) of the lesions, even including those larger than $10 \mathrm{~mm}$, retained their initial size. None of the patients developed gall bladder carcinoma. Two patients showed the largest $(4 \mathrm{~mm})$ increase in lesion size over about 4 years. Because the mean follow up period (2.6 years) was shorter than this, the longer follow up study may be required.

The results in the follow up series also confirmed the diagnostic accuracy of EUS as a means of differentiating polypoid lesions. In
$13 \%$ of the follow up series, the initial EUS detected features characteristic of nonneoplastic lesions which the initial ultrasonography had failed to show. For diagnosing neoplastic lesions, EUS was more specific than ultrasonography, while EUS was as sensitive as ultrasonography. In this study, all patients underwent both ultrasonography and EUS. However, EUS may be unnecessary in patients in whom ultrasonography produces characteristic findings of cholesterol polyp or adenomyomatosis.

Moriguchi and colleagues ${ }^{20}$ reported the natural history of polypoid lesions (presumably benign) during a five year follow up period in 103 patients. ${ }^{20}$ In their study, lesion size did not change in $84 \%$ of patients, although one patient did develop gall bladder carcinoma. Therefore accurate discrimination of nonneoplastic from neoplastic lesions is required before entry into a follow up series. EUS is valuable for managing such potentially malignant polypoid lesions.

Ultrasonographic follow up every six months has been advocated for benign lesions. ${ }^{12}$ 19-21 Regular ultrasonographic surveillance appears to be safer even for non-neoplastic lesions that EUS has initially diagnosed. If subsequent ultrasonography discloses any changes in size or structure of the polypoid lesions, reinvestigation with EUS should be expedited. Cases in which follow up EUS can rule out neoplastic lesions may be appropriate for further careful follow up. As none of the present patients with EUS diagnosis of nonneoplastic lesions developed neoplasia, long term ultrasonographic surveillance may be unnecessary for such patients. This is an important problem to be solved in future.

The present study supports the following principles for management of small polypoid lesions of the gall bladder. Ultrasonography is the preferable modality for screening and following up polypoid lesions. On ultrasonography, polyps smaller than $5 \mathrm{~mm}$ composed of a single tiny echogenic spot and those containing at least a partial aggregation of echogenic spots should be diagnosed as cholesterol polyps. Polypoid lesions in which multiple microcysts or a comet tail artefact are shown indicate adenomyomatosis. Other polypoid lesions should be further examined by EUS. When EUS does not produce such findings in polypoid lesions, neoplasia (adenoma or adenocarcinoma) should be suspected and the lesions should be treated surgically. On the other hand, asymptomatic cholesterol polyps and adenomyomatosis do not require surgery and may be followed up by ultrasonography at intervals of 6-12 months. Changes in size or structure of polypoid lesions on follow up ultrasonography should prompt re-investigation with EUS.

1 Segawa K, Arisawa T, Niwa Y, et al. Prevalence of gall bladder polyps among apparently healthy Japanese: ultrasonographic study. Am f Gastroenterol 1992;87:630-3.

2 Chen CY, Lu CL, Chang FY, et al. Risk factors for gall bladder polyp in the Chinese population. Am $\mathcal{F}$ Gastroenterol

3 Albores-Saavedra J, Henson DE. Tumors of the gall bladder and extrahepatic bile ducts. In: Atlas of tumor pathology, 2nd series, fascicle 22. Washington, DC: Armed Forces Institute of Pathology, 1986:148. 
4 Koga A, Watanabe K, Fukuyama T, et al. Diagnosis and operative indications for polypoid lesions of the gall Arch Surg 1988;123:26-9.

5 Ishikawa $\mathrm{O}$, Ohhigashi $\mathrm{H}$, Imaoka $\mathrm{S}$, et al. The difference in malignancy between pedunculated and sessile polypoid lesions of the gall bladder. Am $\mathcal{F}$ Gastroenterol $1989 ; 84$ 1386-90.

6 Price RJ, Stewart ET, Foley WD, et al. Sonography of polypoid cholesterolosis. AfR Am F Roentgenol 1982;139: $1197-8$.

7 Lichtenstein JE. Adenomyomatosis and cholesterolosis: the "hyperplastic cholesystose". In: Friedman AC, Dachman $\mathrm{AH}$, eds. Radiology of the liver, biliary tract, and pancreas, $1 \mathrm{st}$ ed. St Louis: Mosby, 1994:539-53.

8 Jeffrey RB, Ralls PW. Gall bladder and bile ducts. In: Sonography of the abdomen. New York: Raven Press, 1995:179231 .

9 Sugiyama M, Atomi Y, Kuroda A, et al. Large cholestero polyp of the gall bladder: diagnosis by means of US and polyp of the gall bladder: diagnosis by means

10 Sugiyama M, Xie XY, Atomi Y, et al. Differential diagnosis of small polypoid lesions of the gall bladder: the value of endoscopic ultrasonography. Ann Surg 1999;229:498-504.

11 Weiner SN, Koenigsberg M, Morehouse H, et al. Sonography and computed tomography in the diagnosis of carcinoma of the gall bladder. AfR Am f Roentgenol 1984 142:735-9.
12 Yang HL, Sun YG, Wang Z. Polypoid lesions of the gall bladder: diagnosis and indications for surgery. $\mathrm{Br} \mathcal{F}$ Surg 992;79:227-9.

13 Kubota K, Bandai Y, Noie T, et al. How should polypoid esions of the gall bladder be treated in the era of laparoscopic cholecystectomy? Surgery 1995;117:481-7.

14 Shinkai H, Kimura W, Muto T. Surgical indications for mall polypoid lesions of the gall bladder. Am f Surg 1998; 175:114-17.

15 Hirooka Y, Naitoh Y, Goto H, et al. Differential diagnosis of gall-bladder masses using colour Doppler ultrasonography. F Gastroenterol Hepatol 1996;11:840-6.

16 Furukawa H, Takayasu K, Mukai K, et al. CT evaluation of small polypoid lesions of the gall bladder. HepatoGastroenterol 1995;42:800-10.

17 Yoshimitsu K, Honda H, Kaneko K, et al. Dynamic MRI of the gall bladder: differentiation of benign from malignant. $\mathcal{F}$ Magn Reson Imaging 1997;7:696-701.

18 Kozuka S, Tsubone M, Yasui A, et al. Relation of adenoma to carcinoma in the gall bladder. Cancer 1982;50:226-34.

19 Aldridge MC, Bismuth $\mathrm{H}$. Gall bladder cancer: the polyp-cancer sequence. Br F Surg 1990;77:363-4.

20 Moriguchi $\mathrm{H}$, Tazawa J, Hayashi Y, et al. Natural history of polypoid lesions in the gall bladder. Gut 1996;39:860-2

21 Boulton RA, Adams DH. Gall bladder polyps: when to wait and when to act. Lancet 1997;349:817. 